Research Article

\title{
In Vitro Shoot Regeneration of Oil Seed Crop Sesamum indicum L. from Seedling Cotyledon Explant to Lay Ground for Genetic Transformation in Ethiopia
}

\author{
Micheale Yifter Weldemichael $\left(\mathbb{D},{ }^{1}\right.$ Yemane Tsehaye Bayratsion, ${ }^{2}$ Desta Berhe Sbhatu $\left(\mathbb{D},{ }^{3}\right.$ \\ Girmay Gebresamuel Abraha, ${ }^{4}$ Hagos Mohammedseid Juhar, ${ }^{1}$ Abraha Birhan Kassa, ${ }^{5}$ \\ Fiseha Baraki Sibhatu, ${ }^{6}$ Birhanu Debesay Berhe, ${ }^{7}$ Tagel Elias Sahle, ${ }^{8}$ \\ Mohammed Mebrahtu Mossa, ${ }^{2}$ and Haftay Abadi Gebru ${ }^{7}$
}

${ }^{1}$ Department of Biotechnology, CDANR, Mekelle University, P.O. Box 231, Mekelle, Ethiopia

${ }^{2}$ Department of DCHS, CDANR, Mekelle University, P.O. Box 231, Mekelle, Ethiopia

${ }^{3}$ Department of BCEN, MIT, Mekelle University, P.O. Box 231, Mekelle, Ethiopia

${ }^{4}$ Department of LaRMEP, CDANR, Mekelle University, P.O. Box 231, Mekelle, Ethiopia

${ }^{5}$ Department of Chemistry, CDANR, Mekelle University, P.O. Box 231, Mekelle, Ethiopia

${ }^{6}$ Tigrai Agricultural Research Institute, HuARC, P.O. Box 510, Humera, Ethiopia

${ }^{7}$ Tigrai Biotechnology Center Pvt. Ltd. Co., P.O. Box 223, Mekelle, Ethiopia

${ }^{8}$ Dilla University, P.O. Box 419, Dilla, Ethiopia

Correspondence should be addressed to Micheale Yifter Weldemichael; y.mickye@gmail.com

Received 26 April 2020; Revised 16 May 2020; Accepted 27 May 2020; Published 29 June 2020

Academic Editor: Anshu Alok

Copyright (c) 2020 Micheale Yifter Weldemichael et al. This is an open access article distributed under the Creative Commons Attribution License, which permits unrestricted use, distribution, and reproduction in any medium, provided the original work is properly cited.

\begin{abstract}
This study was conducted to develop an efficient regeneration protocol used for sesame genetic transformation. Published regeneration methods using 6-benzylaminopurine (BAP), indol-3-butyric acid (IBA), and $\alpha$-naphthaleneacetic acid (NAA) were used in this experiment. Cotyledon explants of 14 Ethiopian genotypes collected from Humera Agricultural Research Center (HuARC) were used. Optimized culture conditions were used to investigate the regeneration response of different genotypes. Significant interactions between hormone treatments, MS media, and genotypes for shoot and root regeneration were recorded. Results showed that culture of cotyledons were developed and used as a source of explants for shoot regeneration. The highest shoot number, leaf number, and shoot length were recorded on explants cultured on $1.00 \mathrm{mg} \cdot \mathrm{L}^{-1} \mathrm{BAP}+1.00 \mathrm{mg} \cdot \mathrm{L}^{-1} \mathrm{NAA}$ in Hirhir and Aberghele, $0.75 \mathrm{mg} \cdot \mathrm{L}^{-1} \mathrm{BAP}+1.00 \mathrm{mg} \cdot \mathrm{L}^{-1} \mathrm{NAA}$ in Baha Zeyit, and $1.0 \mathrm{mg} \cdot \mathrm{L}^{-1} \mathrm{BAP}+1.00 \mathrm{mg} \cdot \mathrm{L}^{-1} \mathrm{NAA}$ in Humera 1 , respectively. The lowest shoot number, leaf number, and shoot length were observed on explants cultured on the control in Gondar 1, Borkana, and Baha Necho, Borkana and Beha Necho, and Baha Necho, respectively. Genotypes with well-developed shoots were transferred to root induction media. Under rooting media, the best mean, root number, and root length were observed in Aberghele and ACC44 at $0.1 \mathrm{mg} \cdot \mathrm{L}^{-1}$ IBA and $0.2 \mathrm{mg} \cdot \mathrm{L}^{-1} \mathrm{NAA}$, respectively. Standardizing genotypes trait data to mean 0 and unity variance has helped to group 14 genotypes into four distinct clusters. Hirhir, Abeghele, Baha Zeyit, and Humera 1 were the best genotypes. These findings lay ground to Ethiopian sesame to do further genetic transformation aiming at improving the crop.
\end{abstract}




\section{Introduction}

Sesame (Sesamum indicum L.) is one of the oldest oilseeds crops widely grown in Asia (54.9\%), Africa (40.8\%), and South and Central Americas (4.3\%). India is the leading country and Ethiopia is one of the top 10 sesame producing countries in the world [1]. Sesame is also referred to as the "queen of oilseeds" for the nutritional, pharmacological, and industrial uses of its high-quality oilseeds [2-4]. As a result, sesame is a cash crop and a significant source of income for many countries. Despite its economical, medicinal, and nutritional importance, sesame is considered as an orphan crop as it lags behind other major oil crops in terms of genetic improvement [5]. Hereafter, sesame seed yield capacity is very low compared to other oilseed crops. Likewise, breeding high-yielding sesame varieties is one of the key purposes in sesame production [6]. Hence, the yield remains very low due to a diverse set of adversaries including, but not limited to, drought, salinity, diseases and insect pests, inherent genetic problems (such as seed shattering and indeterminate flowering), and poor agronomic and postharvest handling capabilities. It is, therefore, crucial to systematically investigate the genetic and environmental factors determining sesame yield (quality and quantity) including shattering, drought, and other biotic and abiotic stresses in order to identify and breed genotypes with improved traits.

Traditional crop improvement approaches are very slow and laborious $[7,8]$ and, thus, this fact is open to modern world to utilize advanced genomic and biotechnological tools to accelerate the development of improved sesame genetic resources. Therefore, genetic transformation could be an advantageous alternative [9]. Genetic transformation needs well efficient and reproducible plant tissue culture protocol.

Lack of efficient tissue culture shoot regeneration system further limits genetic engineering of this crop for developing improved lines. Efforts have been made earlier to regenerate sesame in tissue cultures using explants such as shoot tips $[10,11]$, hypocotyls $[12,13]$, cotyledons $[9,14]$, and nodes [15]. Significant success has been achieved for plant regeneration through somatic embryogenesis using hypocotyl or cotyledon as explants $[13,16]$.

The best option for improvement of Sesamum indicum is to transfer genes from other sources through genetic transformation techniques [12]. Due to the production of multiple shoots form preexisting primordial shoot and/or shoot buds, shoot tips and nodes are unsuitable for Agrobacterium-mediated transformation [17]. As a result, there is no proper regeneration system and this leads to no efficient transformation so far [18-21]. Few studies on sesame regeneration in Ethiopian varieties were conducted [22] so far but were unsuitable for transformation. The development of an efficient method of plant regeneration through direct multiple shoot organogenesis from cotyledon explants and the establishment of an optimal selection system is mandatory to do genetic transformation. Hence, this article reports an efficient and reproducible protocol of multiple shoot organogenesis from cotyledon explants of 14
Ethiopian sesames genotypes which is useful for transformation under in vitro conditions. This research work is the first of its kind in Ethiopia and it opened the door for conducting genetic transformation for future genetic improvement.

\section{Materials and Methods}

2.1. Collection and Disinfection of Seeds. The study was carried out at Tigrai Biotechnology Center, Pvt. Ltd. Co. in Mekelle City, northern Ethiopia (Lat.: $13^{\circ} 30^{\prime} 0^{\prime \prime} \mathrm{N}$; Long.: $39^{\circ} 28^{\prime} 11^{\prime \prime}$ E; Alt. 2080 masl) from March 2017 to January 2018. Seeds of 14 sesame genotypes, namely, Setit 1, Setit 2, Humera 1, Hirhir, ADI, ACC44, Baha Necho, Baha Zeyit, Borkena, Gondar 1, Zeri Tesfay, Gumero, Bounji, and Aberghele, were used for this experiment (Figure 1(a)). All genotypes were collected from Humera Agricultural Research Center. Seeds were washed with running tap water for 20 minutes, soaked in 3\% of teepol detergent solution for five minutes, and rinsed with distilled water. Then, seeds were disinfected with $70 \%$ ethanol for 45 seconds at room temperature and rinsed with sterile distilled water three times. Then, seeds were surface-sterilized using sodium hypochlorite solution containing $2 \%$ chlorine for $5 \mathrm{~min}$ followed by a thorough wash in sterile distilled water for 3-4 times. The seeds were then left for soaking in sterile distilled water for $4 \mathrm{~h}$. Aseptic seeds were then blotted on a sterile filter paper, and cotyledons devoid of embryonic axes were dissected from these sterilized seeds with a sterile blade under a biological microscope and transferred to the culture medium.

2.2. Preparations of MS Media Stock Solutions. In the present study, MS basal media as described in [23] were used. $\mathrm{pH}$ was adjusted before autoclaving at 5.8 by suitable addition of $1 \mathrm{~N} \mathrm{HCl}$ and $1 \mathrm{~N} \mathrm{KOH}$ solutions before adding agar $(0.8 \mathrm{w} / \mathrm{v})$ powder for solidification. Culture media were sterilized by autoclaving at $121^{\circ} \mathrm{C}$ temperature with $15^{\circ}$ psi pressure for $15 \mathrm{~min}$. The medium was cooled to $40^{\circ} \mathrm{C}$ before fortifying with filter-sterilized plant growth regulators. The chemicals were weighed using an electronic balance, and all stock solutions were dissolved in sterile distilled water at a ratio of $1 \mathrm{mg} \cdot \mathrm{L}^{-1}$ and kept in plastic bottles in a refrigerator at $4^{\circ} \mathrm{C}$ until used.

\subsection{Preparation of Plant Growth Regulators and Growth} Media. Four concentrations of BAP (6-benzylaminopurine), namely, $0.25,0.5,0.75$, and $1.0 \mathrm{mg} \cdot \mathrm{L}^{-1}$, supplied with $1.00 \mathrm{mg} \cdot \mathrm{L}^{-1}$ of NAA ( $\alpha$-naphthaleneacetic acid) were prepared to supplement shoot regeneration media. Similarly, four concentrations of IBA (indol-3-butyric acid), namely, $0.10,0.20,0.30$, and $0.40 \mathrm{mg} \cdot \mathrm{L}^{-1}$, enriched with $0.20 \mathrm{mg} \cdot \mathrm{L}^{-1}$ of NAA were used to supplement the root induction media. All treatments have controls with no PGRs and were replicated thrice. Culture media were prepared by mixing recommended amounts of MS stock solutions with $3.00 \%$ $(\mathrm{w} / \mathrm{v})$ sucrose as carbon source. The mixed media were divided into 10 parts according to the formulations of PGRs 




(a)

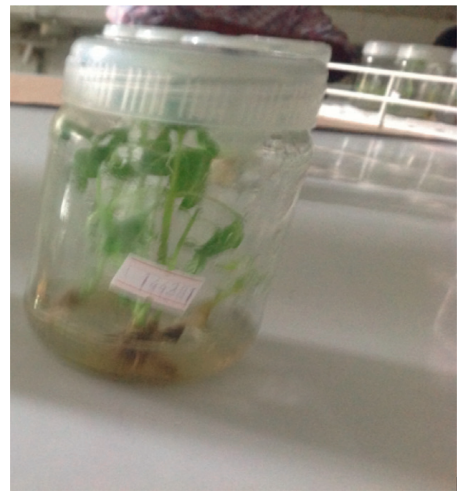

(d)

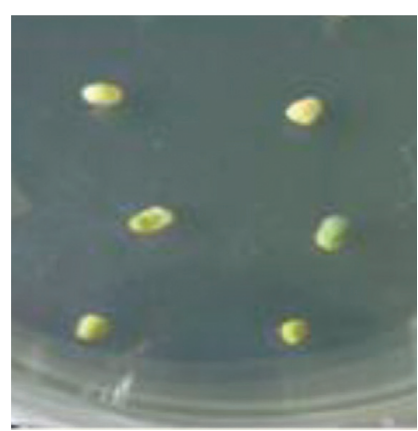

(b)

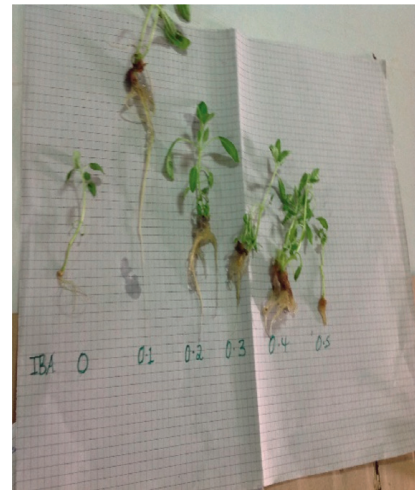

(e)

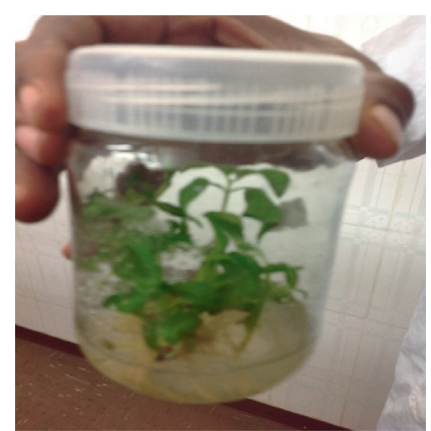

(c)

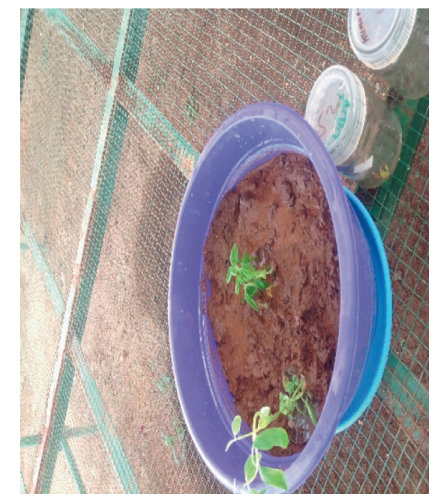

(f)

FIgURe 1: In vitro shoot regeneration in Sesamum indicum genotypes. (a) Seeds of sesame genotypes collected from HuARC. (b) Cotyledon explants after 7 days of culture. (c) Best regeneration of Hirhir genotypes from cotyledon explant. (d) Best shoot number in Aberghele genotype. (e) An in vitro plantlet showing the well-developed root system and make ready for acclimatization. (f) Well-rooted plantlets transferred to soil to acclimatize.

indicated in the treatment design. Five full-strength MS media formulations were put for shoot initiation and five half-strength MS media were put for root induction. The shoot regeneration media were enriched with $0.25 \mathrm{mg} \cdot \mathrm{L}^{-1}$ $\mathrm{BAP}+1.00 \mathrm{mg} \cdot \mathrm{L}^{-1} \quad \mathrm{NAA}, \quad 0.50 \mathrm{mg} \cdot \mathrm{L}^{-1} \quad \mathrm{BAP}+1.00 \mathrm{mg} \cdot \mathrm{L}^{-1}$ $\mathrm{NAA}, \quad 0.75 \mathrm{mg} \cdot \mathrm{L}^{-1} \quad \mathrm{BAP}+1.00 \mathrm{mg} \cdot \mathrm{L}^{-1} \mathrm{NAA}, \quad 1.00 \mathrm{mg} \cdot \mathrm{L}^{-1}$ $\mathrm{BAP}+1.00 \mathrm{mg} \cdot \mathrm{L}^{-1} \mathrm{NAA}$, and no supplement (control). Likewise, the root induction media were enriched with $0.10 \mathrm{mg} \cdot \mathrm{L}^{-1} \quad$ IBA $+0.20 \mathrm{mg} \cdot \mathrm{L}^{-1} \quad \mathrm{NAA}, \quad 0.20 \mathrm{mg} \cdot \mathrm{L}^{-1} \quad$ IBA $+0.20 \mathrm{mg} \cdot \mathrm{L}^{-1} \mathrm{NAA}, 0.30 \mathrm{mg} \cdot \mathrm{L}^{-1} \mathrm{IBA}+0.20 \mathrm{mg} \cdot \mathrm{L}^{-1} \mathrm{NAA}$, $0.40 \mathrm{mg} \cdot \mathrm{L}^{-1} \mathrm{IBA}+0.20 \mathrm{mg} \cdot \mathrm{L}^{-1} \mathrm{NAA}$, and no supplement (control).

2.4. Cotyledon Development and Inoculation for Shoot Regeneration. Cotyledons isolated from the seeds of different varieties of Sesamum indicum were cultured on hormone-free but special media with high amount of sucrose that is 9\% [24]. At the end of the fourth week, data were recorded to calculate the frequency and intensity of adventitious shoot formation from the cultured cotyledons. Cotyledons of each genotype were transferred to each culture bottle, sealed with parafilm, and labeled with media code, date of inoculation, hormone concentration, and name of the genotype plant under investigation. The bottles were transferred into a growth room, randomly arranged on the shelves, and kept in a growth chamber under 16:8 hours (light, dark) photoperiod regime with a light intensity of 2000-2500 lux provided by Mahtab, Iran, $40 \mathrm{~W}$ white bulbs at $25 \pm 2^{\circ} \mathrm{C}$ and $70 \%$ relative humidity (RH). Data on shoot number, shoot length, and leaf number were recorded.

\subsection{Collection of Shoots and Inoculation for Root Proliferation.}

Shoot tips were aseptically collected from three-weeks-old shoots and transferred into half-strength rooting media in Magenta culture bottles under laminar flow cabinet. The bottles were transferred into a growth room, randomly arranged on the shelves, and kept in a growth chamber under continuous light of $2000-2500$ lux at $25 \pm 2^{\circ} \mathrm{C}$ and $70 \%$ RH. Day-to-day observation was carried out to note the rooting responses. Data on root number, root length, and rooting response were recorded.

2.6. Data Analyses. The overall variations on shooting and rooting were partitioned into genotype and PGR main effects as well as interaction. Analyses of genotype and PGR main effects and the interaction component for all data were carried out using ANOVA. In both experiments, the sum square of PGR main effect and the interaction component were partitioned into linear and nonlinear (all possible higher-order polynomials) and tested for significance. GenStat18 software was used to analyze the data. Duncan's multiple range tests at $5 \%$ of significance [25] were used to 
separate treatment means for all combinations. Considering the genotype, treatment, and their interaction components as random, the Best Linear Unbiased Predictor (BLUP) values were estimated. The estimated BLUP values were used as an input for cluster and Mahalanobis $\left(D^{2}\right)$ distance analysis. The $D^{2}$ values obtained from pairs of clusters were considered as the calculated values of chi-square $(\chi 2)$ and were tested for significance at both $1 \%$ and $5 \%$ probability level against the tabulated values of $\chi 2$ for " $P$ "” degree of freedom, where $P$ is the number of characters considered $(P=5)$. After the genotype-trait BLUP data were standardized to mean 0 (zero) and unity variance, and the data matrix was subjected to Hierarchical Cluster Analysis using Ward's Linkage Method and Square Euclidean Distance Measure.

\section{Results and Discussion}

3.1. Cotyledon Development. In this study, sesame seeds were first germinated in the MS media and then cotyledons were excised and used as explants for shooting and rooting protocol development to Ethiopian sesame genotypes. Within one week of culture, the white cotyledons started turning green and became swollen all through, with some of them showing more proliferative activities at the cut ends (Figure 1(b)). By the end of the third week, explants showed appearance of leafy structures developing from the proximal cut end of cotyledons. This developed cotyledon was used as a source of explants for shooting and rooting.

3.2. Shoot Regeneration from Cotyledon Explants. In this study, a total of fourteen genotypes of Sesamum indicum were tested for induction of shoot regeneration. Shoot regeneration responses were studied using four-week cotyledon as explants. The responses were significantly affected by combinations of genotypes and concentration of PGRs. A significant interaction was observed between genotype of the explants and concentration of PGR in shooting (Table 1).

3.2.1. Shoot Number. The best shoot number was recorded on explants cultured on the highest concentration of PGR $\left(1.00 \mathrm{mg} \cdot \mathrm{L}^{-1} \mathrm{BAP}+1.00 \mathrm{mg} \cdot \mathrm{L}^{-1} \mathrm{NAA}\right)$ in Hirhir (12.00) and Aberghele (11.67) genotypes (Figures 1(c) and 1(d)). On the other hand, the lowest was observed on explants cultured at the lowest concentration of PGR $\left(0.25 \mathrm{mg} \cdot \mathrm{L}^{-1} \mathrm{BAP}\right.$ $\left.+1.0 \mathrm{mg} \cdot \mathrm{L}^{-1} \mathrm{NAA}\right)$ in Gandar 1 (1.00), Borkana (1.33), and Baha Necho (1.33) genotypes (Table 2). The result indicated that the highest concentration of BAP with NAA in combination is suitable for adventitious shoot regeneration from the cotyledon cultures of sesame. Similarly, the best shoot number was observed on wild species of Sesamum indicum L. cultured on higher concentration of hormones [26]. In another study, explants cultured on MS media enriched with $2.00 \mathrm{mg} \cdot \mathrm{L}^{-1} \mathrm{BAP}$ and $0.50 \mathrm{mg} \cdot \mathrm{L}^{-1} \mathrm{NAA}$ have yielded the highest number of shoots/callus per explant, as compared to those grown on MS media supplemented with $1.00 \mathrm{mg} \cdot \mathrm{L}^{-1}$ BAP and $0.50 \mathrm{mg} \cdot \mathrm{L}^{-1} \mathrm{NAA}$ [27]. Along similar lines, the authors in [20] reported that the shoot formation response of both explants increased with increasing BA concentration up to $25.0 \mu \mathrm{M}$ and thereafter decreased with further increase in BA concentration. Besides, the highest shoot formation frequency on full-strength MS media produced the highest mean in seedling development from subcultured leaves at $0.1 \mathrm{mg} \cdot \mathrm{L}^{-1}$ kinetin $+0.2 \mathrm{mg} \cdot \mathrm{L}^{-1} \mathrm{NAA}$ [28]. A very recent study [29] reported that the maximum number of young shoots appeared on MS medium supplemented with $0.5 \mathrm{mg} \cdot \mathrm{L}^{-1}$ BAP. This result showed that addition of exogenous PGRs to in vitro culture media is believed to raise the endogenous hormonal level required for triggering flowering and proliferation of many parts of the crop [20]. In this regard, shoot regeneration was significantly influenced by explant type, different hormone types, and concentrations [14]. From our observation, genotypic differences were significant for both the number of explants regenerating shoots and the number of shoots produced per regenerating explant. Furthermore, it is evident that the difference in the frequency of regeneration among different genotypes can be attributed to the difference in the genetic constituents of germplasm.

3.2.2. Leaf Number. Likewise, the genotypes have resulted in varied responses in terms of leaf number in response to variations in PGRs concentrations. Baha Zeyit (62.67) was the best responsive one at $0.75 \mathrm{mg} \cdot \mathrm{L}^{-1} \mathrm{BAP}+1.00 \mathrm{mg} \cdot \mathrm{L}^{-1}$ NAA in terms of mean leaf number. On the other hand, the lowest leaf number was recorded on Borkena (4.67) and Baha Necho (5.33) in the control (Table 3). We have observed that the use of BAP in shooting media is essential. Other workers have attested that BAP was the most effective cytokinin for shoot induction and plant regeneration in Sesamum indicum [20]. Many studies have led to the development and optimization of several protocols with suitable concentrations of PGRs and their combinations that have brought about successful plant regeneration from cultured cells and tissues. Multiple shoot induction was observed in the crop cultured in MS media supplemented with higher concentration of BAP enriched with some NAA [15]. Another similar study of Baskaran and Jayabalan [12] reported that the higher concentration of BAP promoted shoot regeneration in one sesame variety. Similarly, Saravanan and Nadarajan [30] have reported that many sesame varieties respond well to several in vitro culture conditions for callus induction, multiplication, shooting, and rooting and their finding opened new opportunities towards understanding of the regeneration potential for Agrobacterium-mediated gene transformation.

3.2.3. Shoot Length. In vitro regeneration of cotyledon explants was found to be marginally better when it was cultured in MS media supplemented with BAP and NAA. Humera 1 (9.00) showed the best response in mean shoot length with the highest concentration of PGRs (i.e., $1.00 \mathrm{mg} \cdot \mathrm{L}^{-1} \mathrm{BAP}$ and $1.00 \mathrm{mg} \cdot \mathrm{L}^{-1} \mathrm{NAA}$ ) and Baha Necho showed the least mean shoot length in the control (Table 4). The optimum combination of PGRs for adventitious shoot regeneration is found to be at $1.00 \mathrm{mg} \cdot \mathrm{L}^{-1} \mathrm{BAP}$ and NAA. 
TABLE 1: Mean square values of shooting and rooting variables from cotyledon explants.

\begin{tabular}{|c|c|c|c|c|c|c|c|}
\hline \multirow{2}{*}{ Source } & \multirow{2}{*}{$\mathrm{d} f$} & \multicolumn{3}{|c|}{ Full-strength MS shoot variables } & \multicolumn{3}{|c|}{ Half-strength MS rooting variables } \\
\hline & & $\mathrm{LN}$ & SN & SL & RL & $\mathrm{RN}$ & $\mathrm{RR}$ \\
\hline Genotypes (G) & 13 & $787.77^{* *}$ & $61.50^{* *}$ & $11.06^{* *}$ & $24.38^{* *}$ & $214.49^{* *}$ & $1398.80^{* *}$ \\
\hline Treatment $(\mathrm{T})$ & 4 & $904.43^{* *}$ & $99.08^{* *}$ & $21.59^{* *}$ & $15.41^{* *}$ & $1,119.55^{* *}$ & $12,958.30^{* *}$ \\
\hline Linear & 1 & $2,185.15^{* *}$ & $327.72^{* *}$ & $36.02^{* *}$ & $0.47^{\mathrm{ns}}$ & $1,644.19^{* *}$ & $16,922.80^{* *}$ \\
\hline Deviations & 3 & $477.53^{* *}$ & $28.87^{* *}$ & $16.78^{* *}$ & $20.39^{* *}$ & $944.67^{* *}$ & $11,636.80^{* *}$ \\
\hline$G \times T$ & 52 & $149.03^{* *}$ & $7.75^{* *}$ & $2.12^{* *}$ & $10.47^{* *}$ & $265.80^{* *}$ & $1,588.50^{* *}$ \\
\hline Linear & 13 & $171.83^{* *}$ & $12.30^{* *}$ & $3.40^{* *}$ & $14.17^{* *}$ & $136.93^{* *}$ & $1,448.10^{* *}$ \\
\hline Deviations & 39 & $141.42^{* *}$ & $6.24^{* *}$ & $1.70^{* *}$ & $9.24^{* *}$ & $308.75^{* *}$ & $1,635.40^{* *}$ \\
\hline Residual & 140 & 8.77 & 1.31 & 0.92 & 0.87 & 6.49 & 184.20 \\
\hline CV\% & & 15.20 & 20.10 & 18.10 & 26.30 & 20.40 & 24.70 \\
\hline
\end{tabular}

LF: leaf number; SN: shoot number; SL: shoot length; RL: root length; RN: root number; RR: rooting responses. ${ }^{* *} P<0.01$; ${ }^{*} P<0.05$; ns: nonsignificant.

TABLE 2: Interaction effects of PGRs supplements and genotypes on the mean shoot number.

\begin{tabular}{|c|c|c|c|c|c|}
\hline \multirow{2}{*}{ Genotype } & \multicolumn{5}{|c|}{ Treatments } \\
\hline & $\mathrm{T} 1$ & $\mathrm{~T} 2$ & $\mathrm{~T} 3$ & $\mathrm{~T} 4$ & T5 \\
\hline Aberghele & $4.00 \pm 0.00^{1-z}$ & $8.33 \pm 1.53^{\mathrm{d}-\mathrm{g}}$ & $10.33 \pm 1.53^{\mathrm{a}-\mathrm{d}}$ & $6.00 \pm 1.73^{\mathrm{h}-1}$ & $11.67 \pm 1.53^{\mathrm{ab}}$ \\
\hline ACC44 & $2.33 \pm 1.15^{\mathrm{x}-\mathrm{B}}$ & $5.33 \pm 1.15^{\mathrm{j}-\mathrm{q}}$ & $5.33 \pm 1.15^{\mathrm{j}-\mathrm{q}}$ & $9.67 \pm 0.58^{\mathrm{b}-\mathrm{f}}$ & $8.00 \pm 0.00^{\mathrm{e}-\mathrm{h}}$ \\
\hline ADI & $3.00 \pm 1.00^{\mathrm{q}-\mathrm{B}}$ & $4.67 \pm 1.15^{\mathrm{j}-\mathrm{x}}$ & $5.00 \pm 1.00^{\mathrm{j}-\mathrm{v}}$ & $6.33 \pm 2.08^{\mathrm{g}-\mathrm{k}}$ & $4.67 \pm 2.08^{\mathrm{j}-\mathrm{x}}$ \\
\hline Baha Necho & $1.33 \pm 0.58^{\mathrm{AB}}$ & $4.00 \pm 0.00^{1-z}$ & $4.33 \pm 1.53^{\mathrm{k}-\mathrm{y}}$ & $6.67 \pm 1.15^{\mathrm{g}-\mathrm{j}}$ & $4.00 \pm 1.00^{1-z}$ \\
\hline Baha Zeyit & $3.00 \pm 1.73^{\mathrm{q}-\mathrm{B}}$ & $10.67 \pm 0.58^{\mathrm{a}-\mathrm{c}}$ & $6.67 \pm 0.58^{g-j}$ & $9.67 \pm 0.58^{b-f}$ & $8.00 \pm 0.00^{\mathrm{e}-\mathrm{h}}$ \\
\hline Borkena & $1.33 \pm 0.58^{\mathrm{AB}}$ & $3.00 \pm 0.00^{\mathrm{q}-\mathrm{B}}$ & $3.67 \pm 0.58^{1-z}$ & $3.33 \pm 1.53^{\mathrm{o}-\mathrm{A}}$ & $6.00 \pm 1.00^{\mathrm{h}-\mathrm{m}}$ \\
\hline Bounji & $6.67 \pm 1.15^{\mathrm{v}-\mathrm{B}}$ & $5.33 \pm 1.15^{\mathrm{j}-\mathrm{q}}$ & $7.67 \pm 0.58^{\mathrm{f}-\mathrm{i}}$ & $4.00 \pm 0.00^{1-z}$ & $6.67 \pm 1.15^{\mathrm{g}-\mathrm{j}}$ \\
\hline Gondar 1 & $1.00 \pm 0.00^{\mathrm{B}}$ & $3.00 \pm 1.00^{\mathrm{z}-\mathrm{B}}$ & $2.67 \pm 0.58^{\mathrm{v}-\mathrm{B}}$ & $4.00 \pm 0.00^{1-z}$ & $3.33 \pm 0.58^{\mathrm{o}-\mathrm{A}}$ \\
\hline Gumero & $3.67 \pm 0.58^{1-z}$ & $3.67 \pm 0.58^{1-z}$ & $3.67 \pm 0.58^{1-z}$ & $3.00 \pm 1.00^{\mathrm{q}-\mathrm{B}}$ & $4.00 \pm 0.00^{1-z}$ \\
\hline Hirhir & $3.06 \pm 1.00^{\mathrm{q}-\mathrm{B}}$ & $6.00 \pm 1.73^{\mathrm{h}-1}$ & $6.67 \pm 1.53^{\mathrm{g}-\mathrm{j}}$ & $10.67 \pm 1.15^{\mathrm{a}-\mathrm{c}}$ & $12.00 \pm 0.00^{\mathrm{a}}$ \\
\hline Humera 1 & $4.67 \pm 1.15^{\mathrm{j}-\mathrm{x}}$ & $5.67 \pm 1.53^{\mathrm{i}-\mathrm{o}}$ & $11.33 \pm 1.53^{\mathrm{a}-\mathrm{c}}$ & $10.00 \pm 1.73^{\mathrm{a}-\mathrm{e}}$ & $10.33 \pm 1.53^{\mathrm{a}-\mathrm{d}}$ \\
\hline Setit 1 & $4.00 \pm 0.00^{1-\mathrm{z}}$ & $5.33 \pm 1.15^{\mathrm{j}-\mathrm{q}}$ & $5.33 \pm 1.15^{\mathrm{j}-\mathrm{q}}$ & $4.67 \pm 1.15^{\mathrm{j}-\mathrm{x}}$ & $6.00 \pm 2.00^{\mathrm{h}-\mathrm{m}}$ \\
\hline Setit 2 & $4.00 \pm 0.00^{1-\mathrm{z}}$ & $4.00 \pm 0.58^{\mathrm{i}-\mathrm{o}}$ & $4.00 \pm 0.00^{1-z}$ & $4.67 \pm 1.15^{\mathrm{j}-\mathrm{x}}$ & $4.00 \pm 0.00^{1-\mathrm{z}}$ \\
\hline Zeri Tesfay & $5.67 \pm 1.53^{\mathrm{i}-\mathrm{o}}$ & $8.00 \pm 2.00^{\mathrm{e}-\mathrm{h}}$ & $10.00 \pm 2.00^{\mathrm{a}-\mathrm{e}}$ & $9.33 \pm 1.15^{\mathrm{c}-\mathrm{f}}$ & $9.67 \pm 2.08^{b-f}$ \\
\hline
\end{tabular}

Treatments: T1: $0.00 \mathrm{mg} \mathrm{L}{ }^{-1} \mathrm{BAP} \pm 0.00 \mathrm{mg} \cdot \mathrm{L}^{-1} \mathrm{NAA} ; \mathrm{T} 2: 0.25 \mathrm{mg} \cdot \mathrm{L}^{-1} \mathrm{BAP} \pm 1.00 \mathrm{mg} \cdot \mathrm{L}^{-1} \mathrm{NAA} ; \mathrm{T} 3: 0.50 \mathrm{mg} \cdot \mathrm{L}^{-1} \mathrm{BAP} \pm 1.00 \mathrm{mg} \cdot \mathrm{L}^{-1} \mathrm{NAA} ; \mathrm{T} 4: 0.75 \mathrm{mg} \cdot \mathrm{L}^{-1}$ $\mathrm{BAP} \pm 1.00 \mathrm{mg} \cdot \mathrm{L}^{-1} \mathrm{NAA}$; T5: $1.00 \mathrm{mg} \cdot \mathrm{L}^{-1} \mathrm{BAP} \pm 1.00 \mathrm{mg} \cdot \mathrm{L}^{-1} \mathrm{NAA}(\mathrm{CV} \%$ : 20.1; SEM: 0.662 ). Means followed by a different letter indicate significant differences at $P \leq 0.01$.

TABLE 3: Interaction effects of PGRs supplements and genotypes on the mean leaf number.

\begin{tabular}{|c|c|c|c|c|c|}
\hline \multirow{2}{*}{ Genotype } & \multicolumn{5}{|c|}{ Treatments } \\
\hline & $\mathrm{T} 1$ & $\mathrm{~T} 2$ & $\mathrm{~T} 3$ & $\mathrm{~T} 4$ & T5 \\
\hline Aberghele & $13.33 \pm 1.53^{\mathrm{w}-\mathrm{D}}$ & $21.67 \pm 2.08^{\mathrm{k}-\mathrm{r}}$ & $17.33 \pm 3.06^{\mathrm{o}-\mathrm{y}}$ & $19.00 \pm 1.73^{1-\mathrm{w}}$ & $16.00 \pm 2.00^{r-y}$ \\
\hline $\mathrm{ACC} 44$ & $6.33 \pm 2.08^{\mathrm{FG}}$ & $22.67 \pm 5.77^{\mathrm{j}-\mathrm{p}}$ & $26.67 \pm 5.77^{\mathrm{h}-\mathrm{k}}$ & $30.67 \pm 2.31^{\mathrm{e}-\mathrm{h}}$ & $33.33 \pm 4.16^{\mathrm{d}-\mathrm{f}}$ \\
\hline ADI & $9.00 \pm 1.00^{\mathrm{B}-\mathrm{G}}$ & $18.33 \pm 4.73^{\mathrm{m}-\mathrm{x}}$ & $16.67 \pm 2.31^{\mathrm{q}-\mathrm{y}}$ & $22.00 \pm 2.65^{\mathrm{j}-\mathrm{q}}$ & $21.67 \pm 3.51^{\mathrm{k}-\mathrm{r}}$ \\
\hline Baha Necho & $5.33 \pm 3.21^{\mathrm{G}}$ & $16.00 \pm 0.00^{r-y}$ & $9.00 \pm 2.65^{\mathrm{B}-\mathrm{G}}$ & $14.00 \pm 3.46^{\mathrm{p}-\mathrm{y}}$ & $17.00 \pm 3.00^{\mathrm{p}-\mathrm{y}}$ \\
\hline Baha Zeyit & $24.33 \pm 2.08^{\mathrm{i}-1}$ & $33.33 \pm 4.62^{\mathrm{d}-\mathrm{f}}$ & $37.33 \pm 3.21^{\mathrm{cd}}$ & $62.67 \pm 2.31^{\mathrm{a}}$ & $8.33 \pm 2.08^{\mathrm{C}-\mathrm{G}}$ \\
\hline Borkena & $4.67 \pm 1.15^{\mathrm{G}}$ & $9.67 \pm 2.31^{\mathrm{z}-\mathrm{G}}$ & $9.33 \pm 2.89^{\mathrm{A}-\mathrm{G}}$ & $13.00 \pm 2.00^{\mathrm{x}-\mathrm{D}}$ & $15.00 \pm 4.58^{\mathrm{s}-\mathrm{A}}$ \\
\hline Bounji & $6.33 \pm 2.52^{\mathrm{FG}}$ & $20.00 \pm 5.20^{1-\mathrm{u}}$ & $15.00 \pm 1.73^{\mathrm{t}-\mathrm{A}}$ & $12.33 \pm 1.53^{\mathrm{y}-\mathrm{E}}$ & $22.33 \pm 3.51^{\mathrm{j}-\mathrm{q}}$ \\
\hline Gondar 1 & $8.33 \pm 0.58^{\mathrm{C}-\mathrm{G}}$ & $10.00 \pm 1.00^{\mathrm{z}-\mathrm{G}}$ & $13.67 \pm 1.53^{\mathrm{v}-\mathrm{C}}$ & $21.00 \pm 1.00^{\mathrm{k}-\mathrm{s}}$ & $24.00 \pm 1.00^{\mathrm{i}-\mathrm{m}}$ \\
\hline Gumero & $13.33 \pm 1.15^{\mathrm{w}-\mathrm{D}}$ & $14.67 \pm 2.31^{\mathrm{u}-\mathrm{B}}$ & $12.00 \pm 2.00^{y-F}$ & $6.67 \pm 1.15^{\mathrm{e}-\mathrm{g}}$ & $12.00 \pm 0.00^{\mathrm{y}-\mathrm{F}}$ \\
\hline Hirhir & $7.00 \pm 1.00^{\mathrm{E}-\mathrm{G}}$ & $23.33 \pm 4.04^{-\mathrm{n}}$ & $32.33 \pm 4.51^{\mathrm{d}-\mathrm{g}}$ & $39.33 \pm 4.16^{\mathrm{c}}$ & $27.67 \pm 2.52^{\mathrm{g}-\mathrm{j}}$ \\
\hline Humera 1 & $20.67 \pm 1.15^{1-t}$ & $29.33 \pm 5.03^{\mathrm{f}-\mathrm{i}}$ & $35.00 \pm 3.61^{\mathrm{c}-\mathrm{e}}$ & $34.67 \pm 5.69^{c-f}$ & $44.67 \pm 4.16^{\mathrm{b}}$ \\
\hline Setit 1 & $7.67 \pm 1.53^{\mathrm{D}-\mathrm{G}}$ & $19.33 \pm 0.58^{1-\mathrm{v}}$ & $16.67 \pm 2.08^{q-y}$ & $18.33 \pm 2.08^{\mathrm{m}-\mathrm{x}}$ & $17.67 \pm 3.21^{\mathrm{n}-\mathrm{y}}$ \\
\hline Setit 2 & $16.00 \pm 2.00^{\mathrm{r}-\mathrm{y}}$ & $20.67 \pm 1.15^{-\mathrm{o}}$ & $23.00 \pm 3.61^{\mathrm{j}-\mathrm{o}}$ & $22.23 \pm 3.21^{\mathrm{j}-\mathrm{q}}$ & $15.33 \pm 1.15^{\mathrm{s}-\mathrm{z}}$ \\
\hline Zeri Tesfay & $20.00 \pm 3.46^{1-\mathrm{u}}$ & $22.00 \pm 4.00^{\mathrm{j}-\mathrm{q}}$ & $24.67 \pm 3.06^{\mathrm{i}-1}$ & $19.00 \pm 3.61^{1-\mathrm{w}}$ & $20.00 \pm 2.00^{1-\mathrm{u}}$ \\
\hline
\end{tabular}

Treatments: T1: $0.00 \mathrm{mg} \mathrm{L}{ }^{-1} \mathrm{BAP} \pm 0.00 \mathrm{mg} \cdot \mathrm{L}^{-1} \mathrm{NAA} ; \mathrm{T} 2: 0.25 \mathrm{mg} \cdot \mathrm{L}^{-1} \mathrm{BAP} \pm 1.00 \mathrm{mg} \cdot \mathrm{L}^{-1} \mathrm{NAA} ; \mathrm{T} 3: 0.50 \mathrm{mg} \cdot \mathrm{L}^{-1} \mathrm{BAP} \pm 1.00 \mathrm{mg} \cdot \mathrm{L}^{-1} \mathrm{NAA} ; \mathrm{T} 4: 0.75 \mathrm{mg} \cdot \mathrm{L}^{-1}$ $\mathrm{BAP} \pm 1.00 \mathrm{mg} \cdot \mathrm{L}^{-1} \mathrm{NAA}$; T5: $1.00 \mathrm{mg} \cdot \mathrm{L}^{-1} \mathrm{BAP} \pm 1.00 \mathrm{mg} \cdot \mathrm{L}^{-1} \mathrm{NAA}(\mathrm{CV} \%$ : 15.2 ; SEM: 2.418 ). Means followed by a different letter indicate significant differences at $P \leq 0.01$. 
TABLE 4: The interaction effects of PGRs supplements and genotypes on the mean shoot length.

\begin{tabular}{lccccc}
\hline Genotype & & \multicolumn{3}{c}{ Treatments } & \\
& T1 & T2 & T3 & T4 & T5 \\
\hline Aberghele & $4.00 \pm 1.00^{\mathrm{g}-\mathrm{k}}$ & $4.67 \pm 0.58^{\mathrm{e}-\mathrm{j}}$ & $6.67 \pm 0.58^{\mathrm{b}-\mathrm{d}}$ & $4.67 \pm 1.15^{\mathrm{e}-\mathrm{j}}$ & $5.00 \pm 0.00^{\mathrm{d}-\mathrm{i}}$ \\
ACC44 & $3.00 \pm 0.00^{\mathrm{jk}}$ & $5.00 \pm 0.00^{\mathrm{d}-\mathrm{i}}$ & $6.00 \pm 1.73^{\mathrm{b}-\mathrm{f}}$ & $6.33 \pm 1.15^{\mathrm{b}-\mathrm{e}}$ & $4.33 \pm 0.58^{\mathrm{f}-\mathrm{j}}$ \\
ADI & $5.00 \pm 0.00^{\mathrm{d}-\mathrm{i}}$ & $6.33 \pm 1.15^{\mathrm{b}-\mathrm{e}}$ & $5.00 \pm 1.00^{\mathrm{d}-\mathrm{i}}$ & $5.00 \pm 0.00^{\mathrm{d}-\mathrm{i}}$ & $6.00 \pm 1.00^{\mathrm{b}-\mathrm{f}}$ \\
Baha Necho & $2.33 \pm 0.58^{\mathrm{k}}$ & $4.00 \pm 0.00^{\mathrm{g}-\mathrm{k}}$ & $5.00 \pm 2.00^{\mathrm{d}-\mathrm{i}}$ & $5.33 \pm 0.58^{\mathrm{c}-\mathrm{h}}$ & $5.67 \pm 0.58^{\mathrm{b}-\mathrm{g}}$ \\
Baha Zeyit & $4.00 \pm 1.00^{\mathrm{g}-\mathrm{k}}$ & $6.67 \pm 1.53^{\mathrm{b}-\mathrm{d}}$ & $6.33 \pm 0.58^{\mathrm{b}-\mathrm{e}}$ & $6.00 \pm 1.00^{\mathrm{b}-\mathrm{f}}$ & $7.00 \pm 0.00^{\mathrm{bc}}$ \\
Borkena & $4.00 \pm 0.00^{\mathrm{g}-\mathrm{k}}$ & $4.67 \pm 1.15^{\mathrm{e}-\mathrm{j}}$ & $5.33 \pm 0.58^{\mathrm{c}-\mathrm{h}}$ & $4.33 \pm 1.15^{\mathrm{f}-\mathrm{j}}$ & $4.67 \pm 0.58^{\mathrm{e}-\mathrm{j}}$ \\
Bounji & $3.33 \pm 1.53^{\mathrm{i}-\mathrm{k}}$ & $4.00 \pm 0.00^{\mathrm{g}-\mathrm{k}}$ & $4.33 \pm 0.58^{\mathrm{f}-\mathrm{j}}$ & $4.33 \pm 0.58^{\mathrm{f}-\mathrm{j}}$ & $4.67 \pm 0.58^{\mathrm{e}-\mathrm{j}}$ \\
Gondar 1 & $3.00 \pm 1.00^{\mathrm{jk}}$ & $3.67 \pm 1.15^{\mathrm{h}-\mathrm{k}}$ & $6.33 \pm 1.15^{\mathrm{b}-\mathrm{e}}$ & $5.00 \pm 1.73^{\mathrm{d}-\mathrm{i}}$ & $4.33 \pm 0.58^{\mathrm{f}-\mathrm{j}}$ \\
Gumero & $4.67 \pm 1.53^{\mathrm{e}-\mathrm{j}}$ & $4.33 \pm 0.58^{\mathrm{f}-\mathrm{j}}$ & $4.33 \pm 1.15^{\mathrm{f}-\mathrm{j}}$ & $3.67 \pm 0.58^{\mathrm{h}-\mathrm{k}}$ & $4.00 \pm 0.00^{\mathrm{g}-\mathrm{k}}$ \\
Hirhir & $4.00 \pm 1.73^{\mathrm{g}-\mathrm{k}}$ & $6.67 \pm 0.58^{\mathrm{b}-\mathrm{d}}$ & $7.00 \pm 1.00^{\mathrm{bc}}$ & $6.67 \pm 0.58^{\mathrm{b}-\mathrm{d}}$ & $6.33 \pm 0.58^{\mathrm{b}-\mathrm{e}}$ \\
Humera 1 & $5.00 \pm 0.00^{\mathrm{d}-\mathrm{i}}$ & $6.33 \pm 0.58^{\mathrm{b}-\mathrm{e}}$ & $6.33 \pm 0.58^{\mathrm{b}-\mathrm{e}}$ & $7.00 \pm 2.00^{\mathrm{bc}}$ & $9.00 \pm 0.00^{\mathrm{a}}$ \\
Setit 1 & $5.00 \pm 1.00^{\mathrm{d}-\mathrm{i}}$ & $5.67 \pm 1.15^{\mathrm{b}-\mathrm{g}}$ & $7.00 \pm 1.00^{\mathrm{bc}}$ & $6.33 \pm 0.58^{\mathrm{b}-\mathrm{e}}$ & $6.00 \pm 1.73^{\mathrm{b}-\mathrm{f}}$ \\
Setit 2 & $3.00 \pm 1.00^{\mathrm{jk}}$ & $7.33 \pm 0.58^{\mathrm{ab}}$ & $6.67 \pm 1.15^{\mathrm{b}-\mathrm{d}}$ & $6.00 \pm 1.00^{\mathrm{b}-\mathrm{f}}$ & $5.00 \pm 1.00^{\mathrm{d}-\mathrm{i}}$ \\
Zeri Tesfay & $6.67 \pm 0.58^{\mathrm{bcd}}$ & $6.67 \pm 1.53^{\mathrm{b}-\mathrm{d}}$ & $7.33 \pm 1.15^{\mathrm{b}}$ & $5.67 \pm 0.58^{\mathrm{b}-\mathrm{g}}$ & $5.33 \pm 0.58^{\mathrm{c}-\mathrm{h}}$ \\
\hline
\end{tabular}

Treatments: T1: $0.00 \mathrm{mg} \mathrm{L}{ }^{-1} \mathrm{BAP}+0.00 \mathrm{mg} \cdot \mathrm{L}^{-1} \mathrm{NAA} ; \mathrm{T} 2: 0.25 \mathrm{mg} \cdot \mathrm{L}^{-1} \mathrm{BAP}+1.00 \mathrm{mg} \cdot \mathrm{L}^{-1} \mathrm{NAA}$; T3: $0.50 \mathrm{mg} \cdot \mathrm{L}^{-1} \mathrm{BAP}+1.00 \mathrm{mg} \cdot \mathrm{L}^{-1} \mathrm{NAA} ; \mathrm{T} 4: 0.75 \mathrm{mg} \cdot \mathrm{L}^{-1}$ $\mathrm{BAP}+1.00 \mathrm{mg} \cdot \mathrm{L}^{-1} \mathrm{NAA}$; T5: $1.00 \mathrm{mg} \cdot \mathrm{L}^{-1} \mathrm{BAP}+1.00 \mathrm{mg} \cdot \mathrm{L}^{-1} \mathrm{NAA}(\mathrm{CV} \%$ : 18.1; SEM: 0.7828 ). Means followed by a different letter indicate significant differences at $P \leq 0.01$.

BAP is considered to be an effective cytokinin for stimulating shoot length in combination with auxins at different concentrations. Previously, regeneration of sesame was reported in media supplemented with BAP + IAA $[9,14,19]$. In another study, both cytokinins (BAP and TDZ) were used as a good response for shoot length [31]. The present protocol follows direct regeneration of shoots that can lead to the generation of genetically uniform plants which are very important for genetic transformation.

3.3. Root Induction. Varied concentrations of IBA ( 0 to $\left.0.40 \mathrm{mg} \cdot \mathrm{L}^{-1}\right)$ plus constant concentration of NAA $\left(0.20 \mathrm{mg} \cdot \mathrm{L}^{-1}\right)$ have resulted in statistically significant different responses in mean root number, root length, and root initiation response among 14 genotypes tested in this study $(P \leq 0.01)$ (Table 1) (Figure 1(e)).

3.3.1. Root Number. The best mean root number was recorded on explants cultured on $0.20 \mathrm{mg} \mathrm{L}^{-1}$ IBA and constant concentration of NAA $\left(0.20 \mathrm{mg} \mathrm{L}^{-1}\right)$ in Aberghele genotype. On the other hand, the lowest was recorded in Gondar 1 and Humera 1 in the control and Borkana at $0.40 \mathrm{mg} \cdot \mathrm{L}^{-1} \mathrm{IBA}+0.20 \mathrm{mg} \cdot \mathrm{L}^{-1} \mathrm{NAA}$ (Table 5 ). As the concentration of IBA supplement increased to 0.30 and $0.40 \mathrm{mg} \cdot \mathrm{L}^{-1}$, the mean root number tends to decrease with Borkena being the least responsive. The responses of all genotypes in terms of mean root numbers were limited under no PGR application. There are many reports that have shown that combining IBA and NAA were the most effective for rooting in sesame [32, 33]. Similarly, Chamandoosti [34] reports the positive effects of NAA alone on root induction of sesame. Rooting was declined vigorously with increasing the concentration of IBA up to $2.00 \mathrm{mg} \cdot \mathrm{L}^{-1}$. Therefore, NAA is the best PGRs for in vitro rooting in many of plants.

3.3.2. Root Length. Lower IBA concentration (i.e., 0.10 and $0.20 \mathrm{mg} \cdot \mathrm{L}^{-1}$ ) has resulted in relatively better performance in root length. The best mean root length was observed in ACC44 (11.33) with IBA at $0.10 \mathrm{mg} \cdot \mathrm{L}^{-1}+0.20 \mathrm{mg} \cdot \mathrm{L}^{-1} \mathrm{NAA}$. The lowest root length was recorded on Gondar 1, Borkana, and Humera 1 at the control (Table 6).

3.3.3. Root Induction Response. IBA at $0.30 \mathrm{mg} \mathrm{L}^{-1}+$ $0.20 \mathrm{mg} \cdot \mathrm{L}^{-1} \mathrm{NAA}$ resulted in better rooting responses in Borkena and Hirhir, IBA at $0.40 \mathrm{mg} \mathrm{L}^{-1}+0.20 \mathrm{mg} \cdot \mathrm{L}^{-1} \mathrm{NAA}$ in Setit 2, and IBA at $0.10,0.20 \mathrm{mg} \mathrm{L}^{-1}+0.20 \mathrm{mg} \cdot \mathrm{L}^{-1} \mathrm{NAA}$ in ACC44. Humera 1 and Gondar 1 had the lowest rooting response under no IBA and NAA supplements (Table 7). In another recent study, the highest frequency $(65.33 \pm 3.78 \%)$ of root induction was achieved by culturing the elongated shoots in MS medium supplemented with $2.69 \mu \mathrm{M}$ $\alpha$-naphthalene acetic acid (NAA) [35].

3.4. Grouping and Calculating Mahalanobis Distance. Cluster mean values for each trait are given in Table 8 . The 14 genotypes were grouped into four distinct clusters (Figure 2). Cluster I contain five genotypes. Four of the genotypes, namely, Baha Necho, Borkena, Setit 1 , and Setit 2, are released and advanced genotypes with the exception of Aberghele. They are characterized by relatively higher mean root number (13.82), moderate mean root length (3.64), and rooting response (50.41), and above average mean number of shoots and mean shoot length. However, their performance in terms of mean leaf number was below average. Cluster II, on the other hand, includes two local genotypes (i.e., Gumero and Bounji) and one improved genotype (i.e., Gondar 1). The genotypes in this cluster were generally inferior with below-average performance for all the traits compared. Cluster III has one local and three improved genotypes, namely, Zeri Tesfay (local), Humera 1, Hirhir, and Baha Zeyit. They had the highest mean leaf number, shoot number, and shoot length. Genotypes in this cluster have shown moderate performance in the rest of the traits. Finally, cluster IV includes two genotypes, namely, ADI and 
TABLE 5: Interaction effects of IBA and NAA supplements and genotypes on the mean root number.

\begin{tabular}{lccccc}
\hline Genotype & & \multicolumn{3}{c}{ Treatments } & \\
& T1 & $\mathrm{T} 2$ & $\mathrm{~T} 3$ & $\mathrm{~T} 4$ & $\mathrm{~T} 5$ \\
\hline Aberghele & $5.00 \pm 2.00^{\mathrm{x}-\mathrm{B}}$ & $2.00 \pm 1.00^{\mathrm{AB}}$ & $55.00 \pm 5.00^{\mathrm{a}}$ & $4.67 \pm 1.53^{\mathrm{x}-\mathrm{B}}$ & $5.17 \pm 0.76^{\mathrm{w}-\mathrm{A}}$ \\
ACC44 & $3.00 \pm 1.00^{\mathrm{z}-\mathrm{B}}$ & $48.33 \pm 2.89^{\mathrm{d}-\mathrm{i}}$ & $25.00 \pm 5.00^{\mathrm{cd}}$ & $12.33 \pm 2.52^{\mathrm{m}-\mathrm{t}}$ & $15.00 \pm 5.00^{\mathrm{h}-\mathrm{o}}$ \\
ADI & $4.67 \pm 1.53^{\mathrm{x}-\mathrm{B}}$ & $3.67 \pm 1.15^{\mathrm{y}-\mathrm{B}}$ & $13.00 \pm 2.65^{\mathrm{l}-\mathrm{r}}$ & $25.00 \pm 5.00^{\mathrm{cd}}$ & $5.67 \pm 2.08^{\mathrm{w}-\mathrm{A}}$ \\
Baha Necho & $7.00 \pm 1.00^{\mathrm{u}-\mathrm{A}}$ & $9.00 \pm 1.00^{\mathrm{p}-\mathrm{x}}$ & $18.67 \pm 5.03^{\mathrm{e}-\mathrm{h}}$ & $29.00 \pm 3.61^{\mathrm{c}}$ & $26.00 \pm 2.00^{\mathrm{cd}}$ \\
Baha Zeyit & $6.00 \pm 0.00^{\mathrm{v}-\mathrm{A}}$ & $4.00 \pm 0.00^{\mathrm{x}-\mathrm{B}}$ & $7.00 \pm 2.00^{\mathrm{u}-\mathrm{A}}$ & $22.33 \pm 2.52^{\mathrm{d}-\mathrm{f}}$ & $16.67 \pm 2.89^{\mathrm{h}-\mathrm{n}}$ \\
Borkena & $4.00 \pm 0.00^{\mathrm{x}-\mathrm{B}}$ & $13.33 \pm 2.89^{\mathrm{i}-\mathrm{p}}$ & $15.00 \pm 2.00^{\mathrm{h}-\mathrm{o}}$ & $29.33 \pm 1.15^{\mathrm{c}}$ & $0.00 \pm 0.00^{\mathrm{B}}$ \\
Bounji & $7.00 \pm 2.00^{\mathrm{u}-\mathrm{A}}$ & $4.00 \pm 2.00^{\mathrm{x}-\mathrm{B}}$ & $7.67 \pm 1.53^{\mathrm{t}-\mathrm{z}}$ & $17.00 \pm 2.65^{\mathrm{g}-\mathrm{m}}$ & $8.67 \pm 1.53^{\mathrm{p}-\mathrm{y}}$ \\
Gondar 1 & $0.00 \pm 0.00^{\mathrm{B}}$ & $15.00 \pm 5.00^{\mathrm{h}-\mathrm{o}}$ & $6.00 \pm 2.65^{\mathrm{v}-\mathrm{A}}$ & $17.67 \pm 2.52^{\mathrm{g}-\mathrm{m}}$ & $2.00 \pm 0.00^{\mathrm{AB}}$ \\
Gumero & $4.33 \pm 1.53^{\mathrm{x}-\mathrm{B}}$ & $12.67 \pm 2.08^{1-\mathrm{s}}$ & $2.00 \pm 1.00^{\mathrm{AB}}$ & $4.67 \pm 1.53^{\mathrm{x}-\mathrm{B}}$ & $10.67 \pm 1.15^{\mathrm{o}-\mathrm{v}}$ \\
Hirhir & $8.00 \pm 0.00^{\mathrm{s}-\mathrm{z}}$ & $14.67 \pm 3.06^{\mathrm{h}-\mathrm{o}}$ & $11.00 \pm 3.61^{\mathrm{o}-\mathrm{u}}$ & $13.00 \pm 1.00^{\mathrm{l}-\mathrm{r}}$ & $4.33 \pm 0.58^{\mathrm{x}-\mathrm{B}}$ \\
Humera 1 & $0.00 \pm 0.00^{\mathrm{B}}$ & $13.33 \pm 2.89^{\mathrm{i}-\mathrm{p}}$ & $12.67 \pm 2.89^{\mathrm{d}-\mathrm{g}}$ & $18.33 \pm 2.89^{\mathrm{f}-\mathrm{i}}$ & $17.33 \pm 2.52^{\mathrm{g}-1}$ \\
Setit 1 & $3.67 \pm 0.58^{\mathrm{y}-\mathrm{B}}$ & $15.00 \pm 5.00^{\mathrm{h}-\mathrm{o}}$ & $11.67 \pm 2.89^{\mathrm{n}-\mathrm{u}}$ & $14.00 \pm 3.61^{\mathrm{h}-\mathrm{o}}$ & $11.67 \pm 2.08^{\mathrm{n}-\mathrm{u}}$ \\
Setit 2 & $5.00 \pm 0.00^{\mathrm{x}-\mathrm{B}}$ & $10.0 \pm 0.00^{\mathrm{o}-\mathrm{w}}$ & $12.33 \pm 2.52^{\mathrm{m}-\mathrm{t}}$ & $17.00 \pm 2.65^{\mathrm{g}-\mathrm{m}}$ & $23.00 \pm 2.65^{\mathrm{de}}$ \\
Zeri Tesfay & $3.00 \pm 1.00^{\mathrm{z}-\mathrm{B}}$ & $8.33 \pm 0.58^{\mathrm{p}-\mathrm{y}}$ & $25.00 \pm 5.00^{\mathrm{cd}}$ & $18.33 \pm 2.89^{\mathrm{f}-\mathrm{i}}$ & $18.33 \pm 2.89^{\mathrm{f}-\mathrm{i}}$ \\
\hline
\end{tabular}

Means followed by a different letter indicate significant differences at $P \leq 0.01$.

TABLE 6: Interaction effects of IBA and NAA supplements and genotypes on the mean root length.

\begin{tabular}{|c|c|c|c|c|c|}
\hline \multirow{2}{*}{ Genotypes } & \multicolumn{5}{|c|}{ Treatments } \\
\hline & $\mathrm{T} 1$ & $\mathrm{~T} 2$ & T3 & $\mathrm{T} 4$ & T5 \\
\hline Aberghele & $4.00 \pm 2.00^{\mathrm{h}-1}$ & $2.00 \pm 1.00^{\mathrm{m}-\mathrm{p}}$ & $8.00 \pm 1.00^{\mathrm{cd}}$ & $4.33 \pm 1.15^{\mathrm{g}-\mathrm{k}}$ & $3.00 \pm 1.73^{\mathrm{i}-\mathrm{o}}$ \\
\hline ACC44 & $2.67 \pm 0.58^{\mathrm{k}-\mathrm{p}}$ & $11.33 \pm 0.58^{\mathrm{a}}$ & $7.00 \pm 1.00^{\mathrm{de}}$ & $3.67 \pm 1.15^{\mathrm{i}-\mathrm{m}}$ & $6.00 \pm 1.73^{\mathrm{e}-\mathrm{g}}$ \\
\hline ADI & $9.00 \pm 1.00^{\mathrm{bc}}$ & $4.67 \pm 0.58^{\mathrm{f}-\mathrm{j}}$ & $7.00 \pm 0.00^{\mathrm{de}}$ & $3.00 \pm 1.00^{\mathrm{j}-\mathrm{o}}$ & $4.00 \pm 0.00^{\mathrm{h}-1}$ \\
\hline Baha Necho & $2.33 \pm 0.58^{1-p}$ & $2.33 \pm 1.53^{1-p}$ & $3.00 \pm 1.00^{\mathrm{j}-\mathrm{o}}$ & $3.67 \pm 1.15^{\mathrm{i}-\mathrm{p}}$ & $2.33 \pm 0.58^{\mathrm{k}-\mathrm{p}}$ \\
\hline Baha Zeyit & $3.00 \pm 0.00^{\mathrm{j}-\mathrm{o}}$ & $1.00 \pm 0.00^{\mathrm{pq}}$ & $3.33 \pm 0.58^{\mathrm{i}-\mathrm{n}}$ & $3.67 \pm 0.58^{\mathrm{i}-\mathrm{m}}$ & $2.67 \pm 0.58^{\mathrm{k}-\mathrm{p}}$ \\
\hline Borkena & $5.00 \pm 0.00^{\mathrm{f}-\mathrm{i}}$ & $9.67 \pm 0.58^{\mathrm{b}}$ & $4.67 \pm 1.53^{\mathrm{f}-\mathrm{j}}$ & $3.33 \pm 1.15^{\mathrm{i}-\mathrm{m}}$ & $0.00 \pm 0.00^{\mathrm{q}}$ \\
\hline Bounji & $5.00 \pm 1.00^{\mathrm{f}-\mathrm{i}}$ & $2.00 \pm 1.00^{\mathrm{m}-\mathrm{p}}$ & $4.67 \pm 0.58^{f-j}$ & $3.00 \pm 1.00^{\mathrm{j}-\mathrm{o}}$ & $2.00 \pm 1.00^{\mathrm{m}-\mathrm{p}}$ \\
\hline Gondar 1 & $0.00 \pm 0.00^{\mathrm{q}}$ & $3.00 \pm 0.00^{\mathrm{j}-\mathrm{o}}$ & $2.00 \pm 1.00^{\mathrm{m}-\mathrm{p}}$ & $2.00 \pm 0.00^{\mathrm{m}-\mathrm{p}}$ & $1.00 \pm 0.00^{\mathrm{pq}}$ \\
\hline Gumero & $1.33 \pm 0.58^{\mathrm{o}-\mathrm{q}}$ & $3.00 \pm 1.00^{\mathrm{j}-\mathrm{o}}$ & $1.67 \pm 1.15^{\mathrm{n}-\mathrm{q}}$ & $1.67 \pm 0.58^{\mathrm{n}-\mathrm{q}}$ & $3.00 \pm 1.00^{\mathrm{i}-\mathrm{o}}$ \\
\hline Hirhir & $3.00 \pm 0.00^{\mathrm{j}-\mathrm{o}}$ & $2.00 \pm 0.00^{\mathrm{m}-\mathrm{p}}$ & $3.00 \pm 1.00^{\mathrm{j}-\mathrm{o}}$ & $2.33 \pm 1.15^{1-p}$ & $4.00 \pm 0.00^{\mathrm{h}-1}$ \\
\hline Humera 1 & $0.00 \pm 0.00^{\mathrm{q}}$ & $3.33 \pm 1.53^{\mathrm{i}-\mathrm{n}}$ & $4.00 \pm 1.73^{\mathrm{h}-\mathrm{k}}$ & $5.67 \pm 1.15^{\mathrm{e}-\mathrm{h}}$ & $4.67 \pm 1.15^{f-j}$ \\
\hline Setit 1 & $2.00 \pm 0.00^{\mathrm{m}-\mathrm{p}}$ & $2.00 \pm 0.00^{\mathrm{m}-\mathrm{p}}$ & $3.00 \pm 0.00^{j-o}$ & $2.33 \pm 0.58^{1-p}$ & $3.33 \pm 0.58^{\mathrm{i}-\mathrm{n}}$ \\
\hline Setit 2 & $4.00 \pm 1.00^{\mathrm{h}-1}$ & $3.33 \pm 0.58^{\mathrm{i}-\mathrm{m}}$ & $6.33 \pm 1.15^{\mathrm{ef}}$ & $2.00 \pm 1.00^{\mathrm{m}-\mathrm{p}}$ & $5.00 \pm 1.73^{\mathrm{g}-\mathrm{j}}$ \\
\hline Zeri Tesfay & $1.33 \pm 0.58^{\mathrm{o}-\mathrm{q}}$ & $4.33 \pm 1.53^{\mathrm{g}-\mathrm{k}}$ & $5.00 \pm 1.00^{f-j}$ & $3.33 \pm 1.15^{\mathrm{i}-\mathrm{n}}$ & $4.33 \pm 0.58^{\mathrm{g}-\mathrm{k}}$ \\
\hline
\end{tabular}

Treatments: T1: $0.00 \mathrm{mg} \cdot \mathrm{L}^{-1} \mathrm{IBA} \pm 0.00 \mathrm{mg} \cdot \mathrm{L}^{-1} \mathrm{NAA}$; T2: $0.10 \mathrm{mg} \cdot \mathrm{L}^{-1} \mathrm{IBA} \pm 0.20 \mathrm{mg} \cdot \mathrm{L}^{-1} \mathrm{NAA}$; T3: $0.20 \mathrm{mg} \cdot \mathrm{L}^{-1} \mathrm{IBA} \pm 0.20 \mathrm{mg} \cdot \mathrm{L}^{-1} \mathrm{NAA}$; T4: $0.30 \mathrm{mg} \cdot \mathrm{L}^{-1}$ IBA $\pm 0.20 \mathrm{mg} \cdot \mathrm{L}^{-1} \mathrm{NAA}$; T5: $0.40 \mathrm{mg} \cdot \mathrm{L}^{-1}$ IBA $\pm 0.20 \mathrm{mg} \cdot \mathrm{L}^{-1} \mathrm{NAA}(\mathrm{CV} \%: 26.3$; SEM: 0.5404 ). Means followed by a different letter indicate significant differences at $P \leq 0.01$.

TABLE 7: Interaction effects of IBA and NAA supplements and genotypes on rooting response.

\begin{tabular}{|c|c|c|c|c|c|}
\hline \multirow{2}{*}{ Genotype } & \multicolumn{5}{|c|}{ Treatments } \\
\hline & $\mathrm{T} 1$ & $\mathrm{~T} 2$ & $\mathrm{~T} 3$ & $\mathrm{~T} 4$ & $\mathrm{~T} 5$ \\
\hline Aberghele & $23.33 \pm 2.89^{\mathrm{h}-\mathrm{j}}$ & $30.00 \pm 17.32^{\mathrm{f}-\mathrm{i}}$ & $83.33 \pm 14.43^{\mathrm{a}-\mathrm{c}}$ & $41.67 \pm 14.43^{\mathrm{e}-\mathrm{h}}$ & $20.00 \pm 0.00^{\mathrm{hi}}$ \\
\hline $\mathrm{ACC} 44$ & $40.00 \pm 0.00^{\mathrm{e}-\mathrm{h}}$ & $100.00 \pm 0.00^{\mathrm{a}}$ & $100.00 \pm 0.00^{\mathrm{a}}$ & $50.00 \pm 0.00^{\mathrm{d}-\mathrm{f}}$ & $50.00 \pm 0.00^{\mathrm{d}-\mathrm{f}}$ \\
\hline ADI & $75.00 \pm 0.00^{\mathrm{a}-\mathrm{d}}$ & $75.00 \pm 25.00^{\mathrm{a}-\mathrm{d}}$ & $50.00 \pm 25.00^{\mathrm{d}-\mathrm{f}}$ & $83.33 \pm 15.28^{\mathrm{a}-\mathrm{c}}$ & $75.00 \pm 25.00^{\mathrm{a}-\mathrm{d}}$ \\
\hline Baha Necho & $25.00 \pm 0.00^{\mathrm{f}-\mathrm{i}}$ & $72.00 \pm 5.20^{\mathrm{b}-\mathrm{d}}$ & $58.33 \pm 14.43^{\mathrm{c}-\mathrm{e}}$ & $83.33 \pm 14.43^{\mathrm{a}-\mathrm{c}}$ & $23.33 \pm 2.89^{f-i}$ \\
\hline Baha Zeyit & $25.00 \pm 0.00^{\mathrm{f}-\mathrm{i}}$ & $25.00 \pm 0.00^{\mathrm{f}-\mathrm{i}}$ & $75.00 \pm 0.00^{\mathrm{a}-\mathrm{d}}$ & $80.00 \pm 0.00^{\mathrm{a}-\mathrm{c}}$ & $75.00 \pm 0.00^{\mathrm{a}-\mathrm{d}}$ \\
\hline Borkena & $25.00 \pm 0.00^{f-i}$ & $66.00 \pm 0.00^{\mathrm{b}-\mathrm{e}}$ & $75.00 \pm 0.00^{\mathrm{a}-\mathrm{d}}$ & $100.00 \pm 0.00^{\mathrm{a}}$ & $0.00 \pm 0.00^{\mathrm{kl}}$ \\
\hline Bounji & $58.33 \pm 14.43^{\mathrm{c}-\mathrm{e}}$ & $25.00 \pm 0.00^{\mathrm{f}-\mathrm{i}}$ & $50.00 \pm 25.00^{\mathrm{d}-\mathrm{f}}$ & $75.00 \pm 25.00^{\mathrm{a}-\mathrm{d}}$ & $50.00 \pm 25.00^{\mathrm{d}-\mathrm{f}}$ \\
\hline Gondar 1 & $0.00 \pm 0.00^{\mathrm{kl}}$ & $25.00 \pm 0.00^{\mathrm{f}-\mathrm{i}}$ & $75.00 \pm 0.00^{\mathrm{a}-\mathrm{d}}$ & $75.00 \pm 0.00^{\mathrm{a}-\mathrm{d}}$ & $25.00 \pm 0.00^{f-i}$ \\
\hline Gumero & $25.00 \pm 0.00^{\mathrm{f}-\mathrm{i}}$ & $58.33 \pm 14.43^{\mathrm{c}-\mathrm{e}}$ & $25.00 \pm 5.00^{\mathrm{f}-\mathrm{i}}$ & $75.00 \pm 25.00^{\mathrm{a}-\mathrm{d}}$ & $75.00 \pm 25.00^{\mathrm{a}-\mathrm{d}}$ \\
\hline Hirhir & $25.00 \pm 0.00^{\mathrm{f}-\mathrm{i}}$ & $58.33 \pm 14.43^{\mathrm{c}-\mathrm{e}}$ & $75.00 \pm 25.00^{\mathrm{a}-\mathrm{d}}$ & $100.00 \pm 0.00^{\mathrm{a}}$ & $25.00 \pm 0.00^{f-i}$ \\
\hline Humera 1 & $0.00 \pm 0.00^{\mathrm{kl}}$ & $75.00 \pm 25.00^{\mathrm{a}-\mathrm{d}}$ & $75.00 \pm 25.00^{\mathrm{a}-\mathrm{d}}$ & $75.00 \pm 25.00^{\mathrm{a}-\mathrm{d}}$ & $50.00 \pm 25.00^{\mathrm{d}-\mathrm{f}}$ \\
\hline Setit 1 & $25.00 \pm 0.00^{f-i}$ & $50.00 \pm 0.00^{\mathrm{d}-\mathrm{f}}$ & $75.00 \pm 0.00^{\mathrm{a}-\mathrm{d}}$ & $41.67 \pm 14.43^{\mathrm{e}-\mathrm{h}}$ & $50.00 \pm 0.00^{\mathrm{d}-\mathrm{f}}$ \\
\hline Setit 2 & $25.00 \pm 0.00^{f-i}$ & $25.00 \pm 0.00^{\mathrm{f}-\mathrm{i}}$ & $50.00 \pm 25.00^{\mathrm{d}-\mathrm{f}}$ & $75.00 \pm 25.00^{\mathrm{a}-\mathrm{d}}$ & $100.00 \pm 0.00^{\mathrm{a}}$ \\
\hline Zeri Tesfay & $25.00 \pm 0.00^{f-i}$ & $75.00 \pm 25.00^{\mathrm{a}-\mathrm{d}}$ & $91.67 \pm 14.43^{\mathrm{ab}}$ & $66.67 \pm 14.43^{\mathrm{b}-\mathrm{e}}$ & $91.67 \pm 14.43^{\mathrm{ab}}$ \\
\hline
\end{tabular}

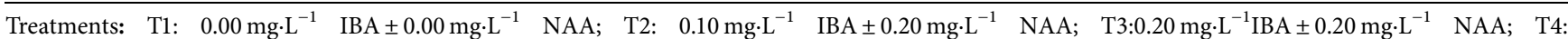
$0.30 \mathrm{mg} \cdot \mathrm{L}^{-1} \mathrm{IBA} \pm 0.20 \mathrm{mg} \cdot \mathrm{L}^{-1} \mathrm{NAA} ; \mathrm{T} 5: 0.40 \mathrm{mg} \cdot \mathrm{L}^{-1} \mathrm{IBA} \pm 0.20 \mathrm{mg} \cdot \mathrm{L}^{-1} \mathrm{NAA}(\mathrm{CV} \%: 24.7$; SEM: 7.836). Means followed by a different letter indicate significant differences at $P \leq 0.01$. 
TABLE 8: Mahalanobis distance between clusters.

\begin{tabular}{lcccr}
\hline Cluster & I & II & III & IV \\
\hline I & - & 19.41 & 49.46 & 28.62 \\
II & & - & 52.70 & 48.91 \\
III & & & - & 31.59 \\
IV & & & - \\
\hline
\end{tabular}

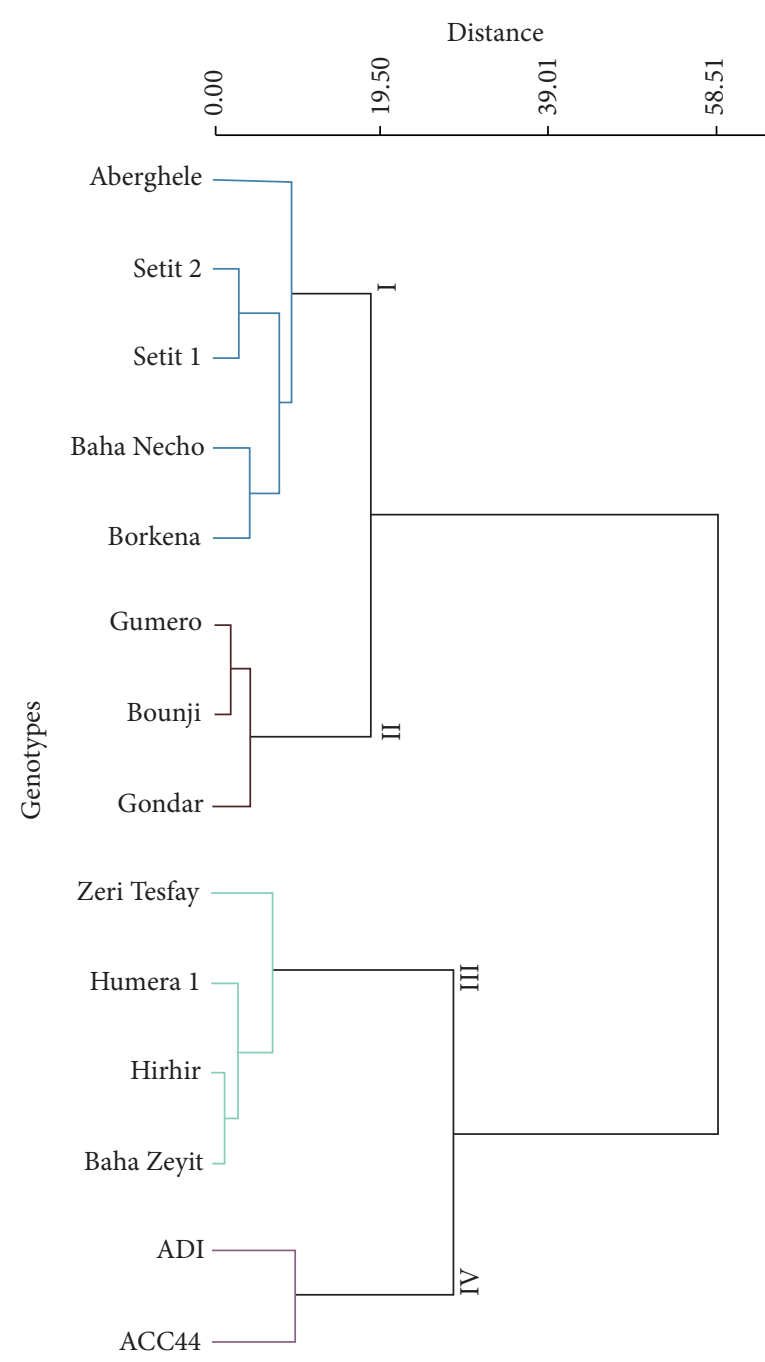

Figure 2: Dendrogram using Ward's method based on dissimilarity matrix of 14 sesame (S. indicum L.) genotypes from the Tigrai Biotechnology Center, Ethiopia.

ACC44. Both genotypes showed high mean performance in most of the traits, especially in root number, root length, and rooting response (Table 9). They have resulted in above average performance in mean shoot number, shoot length, and leaf number.

The genotype grouping observed from the cluster analysis was further confirmed by the Mahalanobis distance analysis among clusters (Table 9). The distance values range from 19.41 (between clusters I and II) to 52.3 (between clusters II and III) and all the distance values were significantly different from each other $(P \leq 0.01)$. Distance values estimated between clusters II and III, I and III, and II and IV were relatively larger than the other variants. The significant difference among clusters as depicted by the Mahalanobis distance would have a breeding implication in sesame improvement programs in Tigrai. The four distinct clusters observed among genotypes signify the differential response of the genotypes to the different PGRs treatments. Genotypes in cluster IV had better performance in root traits (root length, root number, and root response). On the other hand, genotypes under cluster III have superior performance in shoot length, shoot number, and leaf number. The largest distance between clusters I and II with III and cluster II with IV may show a relatively higher divergence among them. Crossing between these clusters may lead to the production of transgressive segregates with better overall performance than either one or both parents. 
TABLE 9: Mean of the quantitative traits for each cluster of sesame genotypes.

\begin{tabular}{lcccccc}
\hline \multirow{2}{*}{ Cluster } & \multicolumn{4}{c}{ Mean of quantitative traits } \\
& Root number & Root length & Root response & Shoot number & Leaf number & Shoot length \\
\hline I & 13.82 & 3.64 & 50.41 & 5.04 & 15.14 & 5.15 \\
II & 8.09 & 2.40 & 48.72 & 3.86 & 4.17 & 28.19 \\
III & 12.53 & 3.21 & 58.83 & 8.00 & 20.72 \\
IV & 15.47 & 5.75 & 67.87 & 5.44 & 5.21 \\
\hline
\end{tabular}

\section{Conclusion}

In this paper, we report an efficient cotyledon-derived direct regeneration protocol of sesame. A reproducible regeneration protocol on Ethiopian sesame genotypes was developed for genetic transformation. In the present study, we established a regeneration system through adventitious shoot formation from excised cotyledons of Sesamum indicum and were able to produce normal healthy plantlets. All fourteen genotypes responded and developed adventitious shoots on the medium were tested. Out of these, Hirhir, Abeghele, Baha Zeyit, and Humera 1 were the best genotypes used for future breeding. Hence, this work will provide a helpful tool for genetic transformation and reproductive growth and for other tissue culture studies. It will also pave the way for efficient Agrobacterium mediated transformation of sesame for desirable traits.

\section{Data Availability}

The datasets used and/or analyzed during the current study are available from the corresponding author on reasonable request.

\section{Conflicts of Interest}

The authors declare no conflicts of interest.

\section{Acknowledgments}

The authors are thankful to Mekelle University and Tigrai Biotechnology Center, Tigrai, Ethiopia, for providing the necessary facilities. The authors are highly indebted to acknowledge Mekelle University for funding this study through Grant no. CRPO/CoDANR/LARGE/001/09.

\section{References}

[1] Food and Agriculture Organization Statistical Databases (FAOSTAT), "FAOSTAT provides free access to food and agriculture data for over 245 countries and territories and covers all FAO regional groupings," 2020, http://faostat.fao. org/.

[2] M.-S. Wu, L. B. B. Aquino, M. Y. U. Barbaza et al., "Antiinflammatory and anticancer properties of bioactive compounds from Sesamum indicum $L$-a review," Molecules, vol. 24, no. 24, p. 4426, 2019.

[3] K. R. Anilakumar, A. Pal, F. Khanum, and A. S. Bawa, "Nutritional, medicinal and industrial uses of sesame (Sesamum indicum L.) seeds-an overview," Agriculturae Conspectus Scientificus, vol. 75, pp. 159-168, 2010.
[4] M. Namiki, "The chemistry and physiological functions of sesame," Food Reviews International, vol. 11, no. 2, pp. 281-329, 1995.

[5] K. Dossa, D. Diouf, L. Wang et al., "The emerging oilseed crop Sesamum indicum enters the "Omics" era," Frontiers in Plant Science, vol. 8, p. 1154, 2017.

[6] H. Zhang, H. Miao, L. Wang et al., "Genome sequencing of the important oilseed crop Sesamum indicumL," Genome Biology, vol. 14, p. 401, 2013.

[7] A. Scheben, F. Wolter, J. Batley, H. Puchta, and D. Edwards, "Towards CRISPR/CAS crops - bringing together genomics and genome editing," New Phytologist, vol. 216, no. 3, pp. 682-98, 2017.

[8] M. Tester and P. Langridge, "Breeding technologies to increase crop production in a changing world," Science, vol. 818, no. 5697, pp. 818-22, 2010.

[9] H. Seo, Y. Kim, T. Park et al., "High-frequency plant regeneration via adventitious shoot formation from deembryonated cotyledon explants of Sesamum indicum L," Vitro Cellular \& Developmental Biology-Plant, vol. 43, pp. 209-214, 2007.

[10] L. George, V. Bapat, and P. Rao, "In vitro multiplication of sesame (Sesamum indicum) through tissue culture," Annals of Botany, vol. 60, no. 1, pp. 17-21, 1987.

[11] J. Lee, Y. Park, Y. Park, and B. Im, "Propagation of sesame (Sesamum indicum L.) through shoot tip culture," Korean Journal of Breeding, 1985.

[12] P. Baskaran and N. Jayabalan, "In vitro mass propagation and diverse callus orientation on Sesamum indicum L.-an important oil plant," Journal of Agricultural Technology, vol. 2, pp. 259-269, 2006.

[13] K. Younghee, "Effects of BA, NAA, 2, 4-D and AgNO3 treatments on the callus induction and shoot regeneration from hypocotyl and cotyledon of sesame (Sesamum indicum L.)," Korean Journal of Horticultural Science and Technology, vol. 42, pp. 70-74, 2001.

[14] B. A. Were, S. Gudu, A. O. Onkware, A. S. Carlsson, and M. Welander, "In vitro regeneration of sesame (Sesamum indicum $L$.) from seedling cotyledon and hypocotyl explants," Plant Cell, Tissue and Organ Culture, vol. 85, pp. 235-239, 2006.

[15] G. Gangopadhyay, R. Poddar, and S. Gupta, "Micropropagation of sesame (Sesamum indicum L.) by in vitro multiple shoot production from nodal explants," 1998.

[16] R. J. Mary and N. Jayabalan, "Influence of growth regulators on somatic embryogenesis in sesame," Plant Cell, Tissue and Organ Culture, vol. 49, pp. 67-70, 1997.

[17] K. R. Rao and K. Vaidyanath, "Biotechnology and improvement of Sesamum," in Proceedings of the National Symposium On Emerging Trends in Plant Tissue Culture and Molecular Biology, Universities Press Ltd., Pune, India, pp. 37-45, November 1999.

[18] S. Chowdhury, A. Basu, and S. Kundu, "A new high-frequency Agrobacterium-mediated transformation technique for 
Sesamum indicum $L$. using de-embryonated cotyledon as explant," Protoplasma, vol. 251, no. 5, pp. 1175-1190, 2014.

[19] A. F. Al-Shafeay, A. S. Ibrahim, M. R. Nesiem, and M. S. Tawfik, "Establishment of regeneration and transformation system in Egyptian sesame (Sesamum indicum L.) cv Sohag 1," GM Crops, vol. 2, no. 3, pp. 182-192, 2011.

[20] M. Yadav, D. Chaudhary, M. Sainger, and P. K. Jaiwal, "Agrobacterium tumefaciens-mediated genetic transformation of sesame (Sesamum indicum L.)," Plant Cell, Tissue and Organ Culture (PCTOC), vol. 103, no. 3, pp. 377-386, 2010.

[21] K. M. Taşkin, A. G. Ercan, and K. Turgut, "Agrobacterium tumefaciens-mediated transformation of sesame (Sesamum indicum L.)," Turkish Journal of Botany, vol. 23, pp. 291-296, 1999.

[22] M. Yifter, D. Sbhatu, F. Mekbib, and E. Abraha, "In vitro regeneration of four ethiopian varieties of sesame (Sesamum indicum L.) using anther culture," Asian Journal of Plant Science, vol. 12, pp. 214-218, 2013.

[23] T. Murashige and F. Skoog, "A revised medium for rapid growth and bio assays with tobacco tissue cultures," Physiologia Plantarum, vol. 15, no. 3, pp. 473-497, 1962.

[24] M. Zimik and N. Arumugam, "Induction of shoot regeneration in cotyledon explants of the oilseed crop Sesamum indicum L." Journal of Genetic Engineering and Biotechnology, vol. 15, no. 2, pp. 303-308, 2017.

[25] D. B. Duncan, "Multiple range and multiple $F$ tests," Biometrics, vol. 11, no. 1, pp. 1-42, 1955.

[26] K. Dasharath, O. Sridevi, P. Salimath, and T. Ramesh, "Production of interspecific hybrids in sesame through embryo rescue," Indian Journal of Crop Science, vol. 2, pp. 193-195, 2007.

[27] M. Sayem, M. Maniruzzaman, S. Siddique, and M. Al-Amin, "In vitro shoot regeneration through anther culture of Brassica spp," Bangladesh Journal of Agricultural Research, vol. 35, pp. 331-341, 2010.

[28] F. M. Jayeoba and O. T. Mustapha, "Effect of hormones on in vitro culture of sesame Sesamum indicum (L.) embryo," Research in Biotechnology, vol. 5, pp. 21-27, 2014.

[29] M. Asad, N. Ahmed, A. Sohail et al., "35. In vitro shoots multiplication from nodal explants of Sesame (Sesamum indicum L.)," Pure and Applied Biology (PAB), vol. 9, pp. 303-308, 2020.

[30] S. Saravanan and N. Nadarajan, "Effect of media supplements on in vitro response of sesame (Sesamum indicum L.) genotypes," Research Journal of Agriculture and Biological Sciences, vol. 1, pp. 98-100, 2005.

[31] N. Shashidhara, H. Ravikumar, N. Ashoka et al., "Thidiazuran (TDZ) induced shoot regeneration in sesame (Sesamum indicum L.)," International Journal of Agriculture, Environment and Biotechnology, vol. 4, pp. 41-44, 2011.

[32] A. Raja and N. Jayabalan, "In vitro shoot regeneration and flowering of sesame (Sesamum indicum L.) cv. SVPR-1," Journal of Agricultural Technology, vol. 7, pp. 1089-1096, 2011.

[33] P. Chakaborti and A. Ghosh, "Variation in callus induction and root-shoot bud formation depend on seed coat of sesame genotypes," Russ J Bot, vol. 5, pp. 14-19, 2010.

[34] F. Chamandoosti, "Influence of plant growth regulators and explant type on multiple shoot induction and somatic embryogenesis in sesame (Sesamum indicum L.)," International Journal of Environmental and Agriculture Research, vol. 2, pp. 68-72, 2016.

[35] A. Debnath, G. Gangopadhyay, D. Basu, and S. Sikdar, "An efficient protocol for in vitro direct shoot organogenesis of Sesamum indicum L. using cotyledon as explant," 3 Biotech, vol. 8, p. 146, 2018. 